\title{
BMJ Open Characteristics of value-based health and social care from organisations' perspectives (OrgValue): a mixed-methods study protocol
}

\author{
Lena Ansmann, ${ }^{1}$ Hendrik Ansgar Hillen, ${ }^{2}$ Ludwig Kuntz, ${ }^{2}$ Stephanie Stock, ${ }^{3}$ \\ Vera Vennedey, ${ }^{3}$ Kira Isabelle Hower, ${ }^{4}$ Cologne Research and Development \\ Network (CoRe-Net)
}

To cite: Ansmann L,

Hillen HA, Kuntz L, et al. Characteristics of value-based health and social care from organisations' perspectives (OrgValue): a mixed-methods study protocol. BMJ Open 2018;8:e022635. doi:10.1136/ bmjopen-2018-022635

- Prepublication history for this paper is available online. To view these files, please visit the journal online (http://dx.doi. org/10.1136/bmjopen-2018022635).

Received 27 February 2018 Revised 11 March 2018 Accepted 13 March 2018

Check for updates

${ }^{1}$ Department of Health Services Research, Faculty of Medicine and Health Sciences, Carl von Ossietzky University Oldenburg, Oldenburg, Germany

${ }^{2}$ Department of Business Administration and Health Care Management, University of Cologne, Cologne, Germany ${ }^{3}$ Institute for Health Economics and Clinical Epidemiology, University Hospital Cologne, Cologne, Germany

${ }^{4}$ Institute of Medical Sociology, Health Services Research, and Rehabilitation Science (IMVR), Faculty of Human Sciences and Faculty of Medicine, University of Cologne, Cologne, Germany

Correspondence to Professor Lena Ansmann; lena.ansmann@uni-oldenburg. de

\section{ABSTRACT}

Introduction Health and social care systems are under pressure to organise care around patients' needs with constrained resources. Several studies reveal that care is constantly challenged by balancing economic requirements against individual patients' preferences and needs. Therefore, value-based health and social care aims to facilitate patient-centredness while taking the resources spent into consideration. The OrgValue project examines the implementation of patientcentredness while considering the health and social care organisations' resource orientation in the model region of the city of Cologne, Germany.

Methods and analysis First, the implementation status of patient-centredness as well as its facilitators and barriers-also in terms of resource orientationwill be assessed through face-to-face interviews with decision-makers (at least $n=18$ ) from health and social care organisations (HSCOs) in Cologne. Second, patients' understanding of patient-centredness and their preferences and needs will be revealed by conducting face-to-face interviews (at least $n=15$ ). Third, the qualitative results will provide the basis for a quantitative survey of decision-makers from all HSCOs in Cologne, which will include questions on patient-centredness, resource orientation and determinants of implementation. Fourth, qualitative interviews with decision-makers from different types of HSCOs will be conducted to develop a uniform measurement instrument on the cost and service structure of HSCOs.

Ethics and dissemination For all collected data, the relevant data protection regulations will be adhered to. Consultation and a positive vote from the ethics committee of the Medical Faculty of the University of Cologne have been obtained. All personal identifiers (eg, name, date of birth) will be pseudonymised. Dissemination strategies include a feedback report as well as research and development workshops for the organisations with the aim of initiating organisational learning and organisational development, presenting results in publications and at conferences, and public relations.

Trial registration number DRKS00011925.
Strengths and limitations of this study

- Mixed-methods study using qualitative interviews of decision-makers and patients as well as a large survey of decision-makers.

- Data collection in a large sample that comprises various types of health and social care organisations in a metropolitan region in Germany.

- Measurement and investigation of the organisational implementation of patient-centred care.

- Further studies would benefit from a linkage of organisational data with patient outcome data.

- Quantitative survey includes decision-makers at the leadership level only and no front-line healthcare providers.

\section{INTRODUCTION}

Recently, it has been suggested to transform care systems to ensure that greater value is placed on the needs of the patients. The concept of value-based care $^{1}$ emphasises that goal transparency and problem-solving capacity are needed in order to overcome problems resulting from the partly conflicting healthcare goals of various actors (eg, profitability vs high quality of care). These partly conflicting goals widen the gap between an increasing demand for services and strained resources within the health and social care system ${ }^{2}$ and call for a redesign of healthcare. Health services research is revealing many deficits in healthcare, which are mostly related to deficits in patient-centred care. ${ }^{3-5}$ According to the Institute of Medicine (IOM), patient-centred care means "Providing care that is respectful of and responsive to individual patient preferences, needs, and values and ensuring that patient values guide all clinical decisions." ${ }^{\prime \prime}$ The IOM states that deficiencies in healthcare quality are always attributable to system problems 
rather than incompetency or misbehaviour of individuals and calls for the examination of the processes and structures required for patient-centred and efficient care.

\section{Patient-centredness and resource orientation in health and social care organisations (HSCOs)}

Various studies evaluating the care of chronically ill patients and vulnerable groups as well as studies analysing management and performance in healthcare ${ }^{347-9}$ reveal that care is constantly challenged by balancing economic requirements against individual patients' preferences and needs. ${ }^{1011}$ HSCOs, like organisations in other fields, naturally strive to maintain and accumulate their resources and prevent resource loss. ${ }^{12} 13$ Organisational resources encompass resources of different types, such as human resources (eg, staff qualification), financial resources (eg, healthcare budget), physical resources (eg, health technology) and information resources (eg, guideline knowledge). In our study, the organisations' orientation towards maintaining, accumulating and preserving their resources is defined as resource orientation. ${ }^{12}$ Due to developments at the societal level, such as demographic change, scarcity of resources in the healthcare system and shortage of skilled staff, HSCOs are increasingly facing difficulties in maintaining their resources. Similarly, they are under pressure to deliver high-quality and patient-centred care. Hence, resource orientation and patient-centredness are oftentimes, but not necessarily, conflicting organisational goals. Whereas, for instance, investments in the qualification of healthcare personnel as a means of accumulating human resources may also be beneficial for patient-centred care, work overload of staff to generate financial savings may hinder patient-centred care.

The interplay between resource orientation and patient-centredness in HSCOs is indicated by a range of studies on organisational and managerial factors in healthcare. ${ }^{14-17}$ Organisational and managerial factors studied with regard to their impact on patient care and patient outcomes include, among others, staff quality, ${ }^{18}$ health literacy of care organisations, ${ }^{19}$ workload and stress $^{20-23}$ as well as social capital. ${ }^{24-26}$ However, patient care has not yet been sufficiently explored in relation to the potentially conflicting goals of patient-centredness and resource orientation in HSCOs. Thus, this study will investigate the implementation of patient-centred care and resource orientation in healthcare organisations.

\section{Aims of the study}

We aim to analyse (1) the patients' and decision-makers' understanding of patient-centred care and organisational resources, (2) HSCOs' coping strategies related to economic and societal developments endangering the organisations' resources, (3) the implementation level of patient-centred and resource-oriented care, and (4) the organisational barriers and facilitators to patient-centred care and resource orientation.

By conducting this study in HSCOs in the city of Cologne-the fourth largest German city with more than 1 million inhabitants-this study will provide valuable information about the current state of patient-centredness and resource orientation in an urban region. Moreover, we hope to identify possible starting points for initiating the redesign of health and social care towards more patient-centredness.

\section{CoRe-Net}

Funded by the German Federal Ministry of Education and Research (BMBF), the 'Cologne Research and Development Network' (CoRe-Net) has been set up since 2017 by various partners from both clinical and social care as well as health services research. ${ }^{27}$ On the basis of three research projects within the city of Cologne, one of which is the OrgValue study, CoRe-Net aims to analyse healthcare delivery for vulnerable patient groups and to establish itself in a sustainable manner. The other two projects within CoRe-Net focus on the one hand on patient trajectories and transitions between different health and social care providers in their last year of life (LYOL-C study) and on the other hand on the trajectories and quality of care of patients with coronary heart disease and mental comorbidity (MenDis-CHD study). The conceptual foundation of the CoRe-Net network relates to the value-based healthcare approach. CoRe-Net aims to facilitate the exchange between academics and healthcare providers within an urban model region and thereby to initiate the redesign of healthcare.

\section{METHODS AND ANALYSIS \\ Study design}

OrgValue is a cross-sectional study integrating mixed methods from qualitative and quantitative social research. The organisations in Cologne that care for the vulnerable patient groups studied in the two accompanying projects within CoRe-Net will be examined using multiple forms of qualitative and quantitative data collection, thus maximising the strengths and minimising the weaknesses of each type of data. ${ }^{28}$ As illustrated in figure 1 , there are four parts of the study.

\section{Sample}

The HSCOs included comprise all organisations caring for the vulnerable patient groups in the city of Cologne, which were studied in the accompanying two projects within CoRe-Net (see table 1).

The preliminary numbers are derived from the administration of the city of Cologne, the Association of Statutory Health Insurance Physicians North Rhine and web-based research (date: November 2017) and will be updated shortly before the conduct of the survey. Since our research objects are HSCOs rather than individual doctors, we will be provided with the numbers and addresses of the single and group practices by the Association of Statutory Health Insurance Physicians North Rhine shortly before the survey. Approximately one-fourth of all private practices are group practices. 


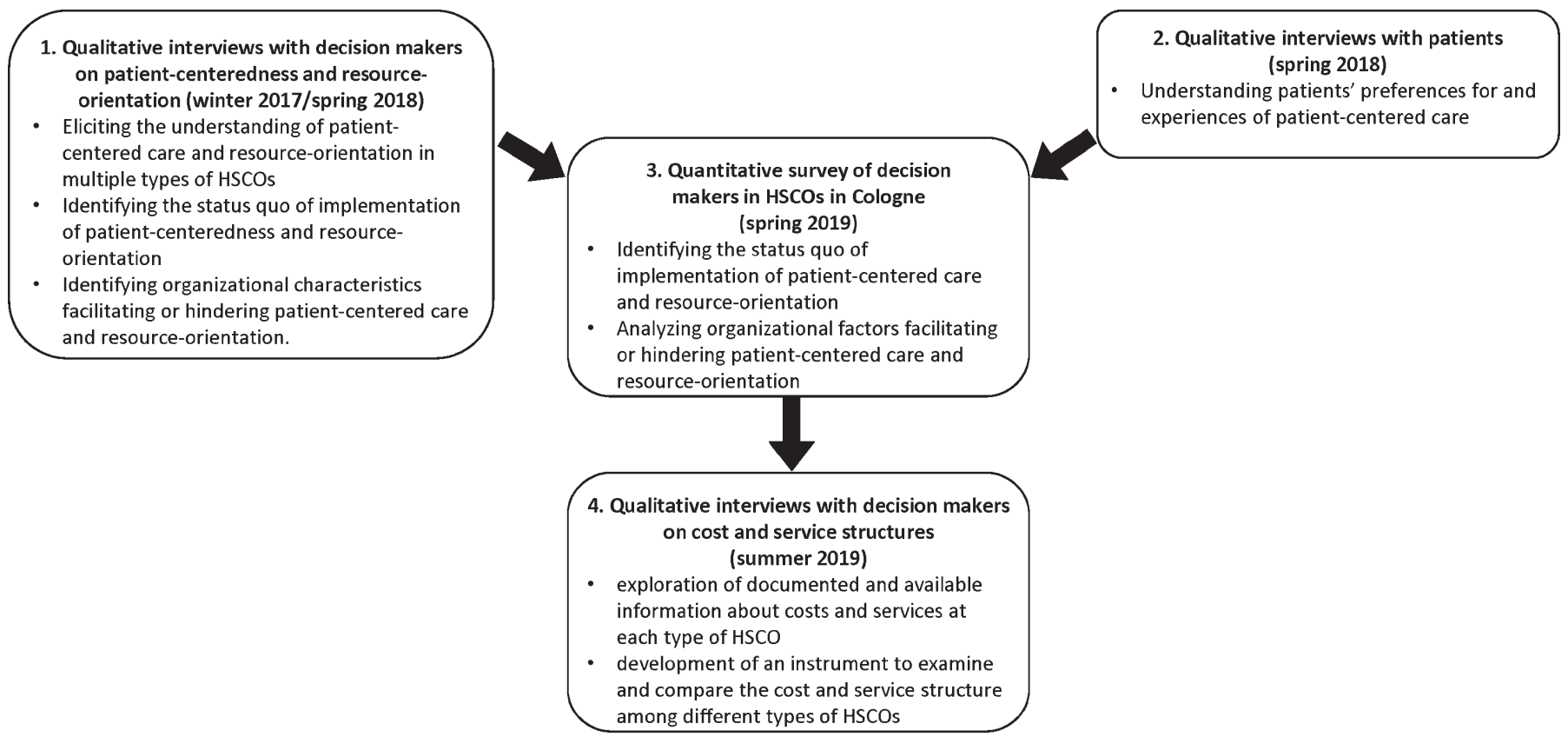

Figure 1 Mixed-methods study design. HSCO, health and social care organisation.

The qualitative interviews in the first part of the study will be conducted with decision-makers from a purposeful sample of those organisations. Decision-makers in our study are defined as clinicians or managers in leading positions with decision-making authority and leadership responsibility within a healthcare organisation. Another inclusion criterion is sufficient German language skills to take part in the survey and interview.

The patients to participate in qualitative interviews during the second part of the study are chronically ill patients in Cologne. Inclusion criteria are a minimum age of 18 years, diagnosis of at least one chronic disease (eg, coronary heart disease, diabetes), sufficient German language skills to take part in the interview and the physical, psychological and cognitive ability to participate in an interview. We aim to obtain a purposeful sample

Table 1 Sample of organisations and doctors working in private practices in the city of Cologne

\section{Type of organisation/doctor in Cologne}

\section{N}

\begin{tabular}{ll}
\hline Hospitals & 22 \\
Long-term outpatient care & 136 Home nursing services \\
& 11 Home hospice services \\
Long-term inpatient care & 100 Nursing homes \\
& 4 Hospices \\
Rehabilitation services & 8 Ambulatory rehabilitation \\
& services \\
& 3 Rehabilitation clinics
\end{tabular}

General practitioners working 870 in private practices

Specialists working in in private 57 Cardiologists practices

88 Psychiatrists

750 Psychotherapists of patients with a variety of chronic illnesses (eg, type of disease, experience in the healthcare system) and personal characteristics (eg, gender, age).

The quantitative survey in the third part of the study will be conducted with decision-makers in all aforementioned organisations. Interviewees to be involved in part four of the study are decision-makers with knowledge about the cost structure and service portfolio of the respective organisation.

\section{Recruitment}

In part one of our study, interviews with decision-makers from different types of organisations will be conducted according to the concept of theoretical sampling (at least $\mathrm{n}=18) .{ }^{29}$ Potential participants will be approached via the original CoRe-Net networking structure and via the new acquisition of participating organisations within the city of Cologne.

For the patient interviews in part two, a minimum of 15 interviews with patients will be conducted. If additional information can be expected, further interviews will be conducted until theoretical saturation is reached. ${ }^{30}$ Potential participants will be contacted via the network of practice partners as well as posters and flyers distributed in public places and newspaper advertisements.

For the survey study in part three, participants from all HSCOs of interest in the city of Cologne will be recruited. Recruitment will take place based on the contact information of HSCOs gathered from the administration of the city of Cologne, the Association of Statutory Health Insurance Physicians North Rhine, and web-based search.

To recruit participants for the qualitative study of costs and services in part four, a single item in the quantitative survey asks about (1) willingness to participate in a subsequent qualitative interview and (2) eligible key 
informants to approach. Those key informants will be contacted in order to arrange the interviews.

\section{Measures}

The semi-structured qualitative interview guide for the decision-maker interviews in part one focuses on main topics derived from literature and a workshop with partners from practice. Each topic is operationalised by core questions and narrative-generating subquestions. The deductively derived categories can later be complemented and modified by inductively identified categories. In all three qualitative data collections, we will pursue an iterative process of interviewing, continuously analysing the data and refining the interview guide for subsequent interviews. Core questions for the interviews in study part one are:

1. How do decision-makers define patient-centred care?

2. What hinders or facilitates the implementation of patient-centred care in their organisations?

3. How do organisations deal with their resources and what resources are needed/lacking?

4. To what extent do organisations assure continuity of care throughout the care pathway and in collaboration with other organisations and providers?

The semi-structured qualitative interview guide for the patient interviews in part two focuses on the question of how patients characterise patient-centred care in comparison to existing conceptual models of patient-centred care derived from the scientific literature and expert views. The topic is operationalised by core questions and narrative-generating subquestions.

The quantitative survey of decision makers in HSCOs in part three will use a questionnaire that is to be developed on the basis of relevant literature and after the interviews with decision-makers have been analysed. Thus, the hypotheses and issues of relevance explored in phase one can be tested with a specific questionnaire. The selection of validated instruments is guided by international frameworks on organisational determinants of patient-centredness and implementation research. Literature examining the implementation of patient-centred care ${ }^{31} 32$ captures organisational dimensions such as leadership, a strategic vision, the involvement of patients and families, a supportive work environment as well as measurement and feedback as critical determinants of patient-centred care in HSCOs. The Consolidated Framework for Implementation Research by Damschroeder ${ }^{33}$ proposes several organisational factors ('inner setting') that facilitate or hinder the effective implementation of interventions in healthcare, which can also be relevant for the development of patient-centred care. These organisational factors are structural characteristics, networks and communication, culture and implementation climate. Since the survey development is based on the qualitative interview results in addition to the literature, the selection of instruments cannot be presented at this stage.

The semi-structured qualitative interview guide for the decision-maker interviews within the fourth part focuses on the exploration of documented and available information about costs and services at each type of HSCO under consideration. Moreover, existing key performance indicators will be identified and assessed regarding their usefulness for comparisons among different types of HSCOs. The topic is operationalised by core questions and narrative-generating subquestions.

\section{Data collection}

In study part one, the qualitative semistructured interviews with decision-makers in HSCOs will be conducted at a place of the participants' choice (eg, home, healthcare organisation, researchers' institute) and will last about 1 hour. ${ }^{34}$ Each interview will be audiotaped and transcribed according to established standards. ${ }^{35}$

In study part two, either individual interviews or focus group interviews will be chosen for the qualitative interview study with chronically ill patients, depending on the patients' physical and psychological abilities and preferences for participation in individual or group interviews. Previous experiences of patient-centred care and expectations will be discussed to define the most patient-relevant dimensions of patient-centred care. The interview guide will be adapted if necessary throughout the interviews. All interviews will be audiotaped and transcribed according to established standards. ${ }^{35}$

The method used for surveying decision-makers of HSCOs in study part three is classified in the literature as 'key informant survey' and is frequently used in order to assess the knowledge of employees who generally have decision-making authority. ${ }^{36}$ In our case, clinical and managerial decision-makers possess valuable information about core structures and processes within the organisation. Key person surveys enable a substantially larger number of organisations to be surveyed at lower cost. The person or steering board in the highest position within each organisation will in a first approach be contacted by post with study information accompanied by an informed consent form, the postal survey and two return envelopes. Standards of survey development ${ }^{30}$ 37-39 will be followed, and the questionnaire will be pretested with established methods. ${ }^{40}$ The survey will be conducted according to Dillman's Total Design Method, with two personalised reminders being sent out. ${ }^{41}$ Furthermore, several strategies shown to increase response rates will be applied (eg, personalised letters, academic origin of the study). ${ }^{42} \mathrm{~A}$ donation of $€ 1$ per responding decision-maker, survey feedback via anonymous benchmarking reports ${ }^{43}$ and the prospect of outreach events organised by CoRe-Net serve as incentives for participation.

In study part four, qualitative semistructured interviews with decision-makers in HSCOs will be used as a method to explore the cost and service structures as well as key performance indicators within each type of HSCO (at least $n=6$ ). The face-to-face interviews will be conducted at a place of each participant's choice (eg, home, healthcare organisation, researchers' institute) and will last no longer than $90 \mathrm{~min}$. Memory protocols will be used. 


\section{Data analysis}

The transcripts of interviews with decision-makers and patients in phases one and two of our study will be analysed by two multidisciplinary independent researchers using established methods of content analysis ${ }^{44}$ and will subsequently be interpreted by a group of researchers. Inductively identified categories can then complement and modify the deductively derived categories. The results will be used to inform survey development as well as to generate hypotheses regarding which organisational characteristics impact the delivery of patient-centred care.

The survey data preparation will be conducted with the Teleform ${ }^{\odot}$ software, which is a software for data capturing. Afterwards, plausibility tests will be run. Data from already validated scales in the survey will be constructed according to the coding manuals after demonstrating the psychometric properties. Data from self-developed instruments on measured constructs will be psychometrically analysed for their factorial validity and reliability before scales will be built. After conducting descriptive analyses stratified by organisational type, regression analyses and structural equation modelling will be performed in order to examine associations between organisational structures and processes and the self-reported implementation of patient-centred and resource-oriented care within the healthcare organisations under study. Using subgroup and cluster analyses, types of organisations may be explored.

The decision-maker interviews in part four will be documented in memory protocols, which will be used to derive ideas for the development of an assessment tool that captures costs, the service portfolio and key performance indicators that are specific to each organisational type. Additionally, the response to the survey item on the respondents' willingness to participate in subsequent interviews of our study will be analysed since it reveals valuable information on the organisations' willingness to provide organisational data for research.

\section{Patient and public involvement}

As the main target group, decision-makers from health and social care organisations are involved in the planning of the study design, recruitment and instrument development via workshops throughout the study duration. Patients will be recruited for interviews to assess their preferences and expectations. Moreover, CoRe-Net explicitly involves providers, decision-makers, patient representatives and the public in the development of the three studies via public relations as well as research and development workshops.

\section{ETHICS AND DISSEMINATION \\ Ethical considerations}

For all collected data, the relevant data protection regulations will be adhered to. Consultation and a positive vote have been obtained from the ethics committee of the Medical Faculty of the University of Cologne. All participants in this study will receive written information about the aims and procedures of the study and will be asked for written consent to collect, analyse and save their data. All personal identifiers will be pseudonymised.

\section{Dissemination plan}

First, feedback on the survey results will be given to the respondents in form of a report with the aim to initiate organisational learning and development. The report will cover results of the complete sample of organisations, stratified by organisational types. Moreover, all participating organisations will receive an additional report showing their results in comparison to aggregated scores of all organisations and especially organisations of the same type.

Second, tools for the self-assessment of HSCOs with regard to patient-centredness and resource orientation will be developed. Thirdly, 'benchmark' workshops will be conducted, in which decision-makers can on the one hand share their best-practice strategies in relation to patient-centredness and resource orientation based on the survey results and on the other hand learn about instruments for promoting organisational development. Fourthly, graphically illustrated and easy-to-read fact sheets for decision-makers with evidence-based and pragmatic measures facilitating patient-centredness in HSCOs will be delivered.

The results and deliverables will be disseminated within the region of Cologne via the Cologne lab conferences and CoRe-Net public relations. National dissemination will be achieved by informing policy-makers, health insurance funds and professional organisations via conferences and seminars (eg, Spring School of the German Network for Health Services Research). Dissemination within the national and international scientific community performing organisational health services research will take place through scientific publications, conferences and discussions within working groups of scientific associations, for example, the Academy Health Conference on the Science of Dissemination and Implementation.

Contributors All members designed the study. LA drafted and revised all sections of the paper and is guarantor. SS and LK revised the paper. LA, KIH, HH and VV designed data collection tools, defined the sampling criteria and revised the paper.

Funding This work was supported by the German Federal Ministry of Education and Research (grant no. 01GY1606).

Competing interests None declared.

Patient consent Not required.

Ethics approval Ethics committee of the Medical Faculty of the University of Cologne.

Provenance and peer review Not commissioned; peer reviewed for ethical and funding approval prior to submission.

Open Access This is an Open Access article distributed in accordance with the Creative Commons Attribution Non Commercial (CC BY-NC 4.0) license, which permits others to distribute, remix, adapt, build upon this work non-commercially, and license their derivative works on different terms, provided the original work is properly cited and the use is non-commercial. See: http://creativecommons.org/ licenses/by-nc/4.0/

(c) Article author(s) (or their employer(s) unless otherwise stated in the text of the article) 2018. All rights reserved. No commercial use is permitted unless otherwise expressly granted. 


\section{REFERENCES}

1. Porter ME, Pabo EA, Lee TH. Redesigning primary care: a strategic vision to improve value by organizing around patients' needs. Health Aff 2013;32:516-25.

2. Gray M. Value based healthcare. BMJ 2017;356:j437.

3. van Deen WK, Esrailian E, Hommes DW. Value-based health care for inflammatory bowel diseases. J Crohns Colitis 2015;9:421-7.

4. Couët N, Desroches S, Robitaille H, et al. Assessments of the extent to which health-care providers involve patients in decision making: a systematic review of studies using the OPTION instrument. Health Expect 2015;18:542-61.

5. Elwyn G, Frosch DL, Kobrin S. Implementing shared decisionmaking: consider all the consequences. Implement Sci 2016;11:114.

6. Institute of Medicine. Crossing the quality chasm: a new health system for the 21st century. Washington, 2001.

7. Gaertner J, Drabik A, Marschall U, et al. Inpatient palliative care: a nationwide analysis. Health Policy 2013;109:311-8.

8. Lamb BW, Brown KF, Nagpal K, et al. Quality of care management decisions by multidisciplinary cancer teams: a systematic review. Ann Surg Oncol 2011;18:2116-25.

9. Rijckmans MJ, Garretsen HF, van de Goor LA, et al. Key concepts of demand-driven health care; an approach based on client's needs. Med Law 2005;24:463-77.

10. Fisher ES, Wennberg JE, Quality HC. Health care quality, geographic variations, and the challenge of supply-sensitive care. Perspect Biol Med 2003;46:69-79.

11. Wennberg JE. Unwarranted variations in healthcare delivery: implications for academic medical centres. BMJ 2002;325:961-4.

12. Pfeffer J, Salancik GR. The external control of organizations: a resource dependence perspective. $1978^{\text {th }}$ edn. Stanford CA: Stanford Business Books, 2003.

13. Yeager VA, Menachemi N, Savage GT, et al. Using resource dependency theory to measure the environment in health care organizational studies: a systematic review of the literature. Health Care Manage Rev 2014;39:50-65.

14. Hearld LR, Alexander JA, Fraser I, et al. Review: How do hospital organizational structure and processes affect quality of care?: a critical review of research methods. Med Care Res Rev 2008;65:259-99.

15. Sunol R, Wagner C, Arah OA, et al. Evidence-based organization and patient safety strategies in European hospitals. Int J Qual Health Care 2014;26(Suppl 1):47-55.

16. Halligan M, Zecevic A. Safety culture in healthcare: a review of concepts, dimensions, measures and progress. BMJ Qual Saf 2011;20:338-43.

17. Scott RA, Aiken LH, Mechanic D, et al. Organizational aspects of caring. Milbank Q 1995;73:77-95.

18. Kuntz L, Sülz S. Treatment speed and high load in the emergency department-does staff quality matter? Health Care Manag Sci 2013;16:366-76.

19. Kowalski C, Lee SY, Schmidt A, et al. The health literate health care organization 10 item questionnaire (HLHO-10): development and validation. BMC Health Serv Res 2015;15:47.

20. Ansmann L, Kowalski C, Ernstmann N, et al. Do breast cancer patients receive less support from physicians in German hospitals with high physician workload? A multilevel analysis. Patient Educ Couns 2013;93:327-34

21. Kuntz L, Mennicken R, Scholtes S. Stress on the ward: evidence of safety tipping points in hospitals. Manage Sci 2015;61:754-71.

22. Tarnow-Mordi WO, Hau C, Warden A, et al. Hospital mortality in relation to staff workload: a 4-year study in an adult intensive-care unit. The Lancet 2000;356:185-9.
23. Montgomery VL. Effect of fatigue, workload, and environment on patient safety in the pediatric intensive care unit. Pediatr Crit Care Med 2007;8(2 Suppl):S11-S16.

24. Ernstmann N, Driller E, Kowalski C, et al. Social capital and quality emphasis: a cross-sectional multicenter study in German hospitals. Int J Healthc Manag 2012;5:98-103.

25. Ansmann L, Wirtz M, Kowalski C, et al. The impact of the hospital work environment on social support from physicians in breast cancer care. Patient Educ Couns 2014;96:352-60.

26. Van Bogaert $P$, Timmermans $O$, Weeks $S M$, et al. Nursing unit teams matter: Impact of unit-level nurse practice environment, nurse work characteristics, and burnout on nurse reported job outcomes, and quality of care, and patient adverse events - a cross-sectional survey. Int J Nurs Stud 2014;51:1123-34.

27. Karbach U, Ansmann L, Scholten N, et al. Report from an ongoing research project: The Cologne Research and Development Network (CoRe-Net) and the value-based approach to healthcare. Zeitschrift für Evidenz, Fortbildung und Qualität im Gesundheitswesen 2017.

28. Klassen AC, Creswell J, Plano Clark VL, et al. Best practices in mixed methods for quality of life research. Qual Life Res 2012;21:377-80.

29. Glaser BG, Strauss AL. Grounded theory: strategien qualitativer Forschung. 2nd edn. Bern: Huber, 2008.

30. Schaeffer NC, Dykema J. Questions for surveys: current trends and future directions. Public Opin Q 2011;75:909-61.

31. Luxford K, Safran DG, Delbanco T. Promoting patient-centered care: a qualitative study of facilitators and barriers in healthcare organizations with a reputation for improving the patient experience. Int J Qual Health Care 2011;23:510-5.

32. Shaller D. Patient-centered care: what does it take? The Commonwealth Fund, 2007.

33. Damschroder LJ, Aron DC, Keith RE, et al. Fostering implementation of health services research findings into practice: a consolidated framework for advancing implementation science. Implement Sci 2009;4:50.

34. Waller V, Farquharson K, Dempsey D. Qualitative social research: contemporary methods for the digital age. Los Angeles: Sage, 2016.

35. Flick U, Kvale S, Angrosino MV, et al. eds. The Sage qualitative research kit. London: Sage, 2007.

36. Rousseau DM. Assessing organizational culture: the case of multiple methods. Schneider B, ed. Organizational climate and culture. San Francisco, Calif: Jossey-Bass, 2010:153-92.

37. Poggie J. Toward quality control in key informant data. Hum Organ 1972;31:23-30.

38. Bradburn NM, Sudman S, Wansink B. Asking questions: the definitive guide to questionnaire design-for market research, political polls, and social and health questionnaires. Hoboken: John Wiley \& Sons Inc, 2004.

39. Schaeffer NC, Presser S. The science of asking questions. Annu Rev Sociol 2003;29:65-88.

40. Prüfer $P$, Rexroth $M$. Zwei Phasen-Pretesting. In: Mohler PP, Lüttinger P, Querschnitt: Festschrift für Max Kaase. Mannheim: ZUMA, 2000:203-19

41. Dillman DA. Mail and telephone surveys: the total design method: a Wiley-Interscience publication. New York: Wiley, 1978.

42. Edwards PJ, Roberts I, Clarke MJ, et al. Methods to increase response to postal and electronic questionnaires. Cochrane Database Syst Rev 2009;3:MR000008.

43. Ivers N, Jamtvedt G, Flottorp S, et al. Audit and feedback: effects on professional practice and healthcare outcomes. Cochrane Database Syst Rev 2012;6:CD000259.

44. Miles MB, Huberman AM, Saldaña J. Qualitative data analysis: a methods sourcebook. $3^{\text {rd }}$ edn. Los Angeles, California: Sage Publications, 2014 